\title{
Challenges of Meeting Surgical Needs in the Developing World
}

\author{
Richard A. Gosselin • Yaw-Adu Gyamfi • \\ Sandro Contini
}

Published online: 23 November 2010

(C) The Author(s) 2010. This article is published with open access at Springerlink.com

\begin{abstract}
The burden of surgical conditions and diseases is increasing in low-income and middle-income countries, but the capacity to meet the demands they present is not following pace. Ongoing initiatives, such as brief visits by surgeons from advantaged countries, sending surgical residents to spend time in a developing country as part of their training, or ships weighing anchor offshore and offering some limited on-shore or on-board services, have not proven successful. More comprehensive and sustainable solutions include the development of local training programs, better retention of trainees with adequate incentives particularly in rural areas, and engaging government and professional associations, as well as academic institutions, to develop and implement policies to address local training needs.
\end{abstract}

\section{Introduction: burden of surgical disease}

Although there is increasing awareness of the importance of unmet needs for surgical care worldwide, data for resource-poor environments remain woefully scarce [1]. It is estimated that up to half the world's population lacks access to basic surgical care [2]. Low-income and middle-

\footnotetext{
R. A. Gosselin ( $\square)$

Department of Orthopedic Surgery, University of California San Francisco, San Francisco, California, USA

e-mail: froggydoc@comcast.net

Y.-A. Gyamfi

Anesthesia Department, Ghana Medical School, Accra, Ghana

S. Contini

Department of Surgical Sciences, University of Parma, Parma, Italy
}

income countries (LMICs) are often bunched together in the literature but, in fact, have quite different economic profiles. The World Bank defined, in 2009 US dollars, a low-income country as having a per-capita gross national income (GNI) of $\$ 995$ or less and a middle-income country as having a per-capita GNI of $\$ 996$ to $\$ 3945$.

Economic development and industrialization are increasing the life expectancy in LMICs and thus also the incidence and prevalence of chronic noncommunicable diseases that often require surgical treatment (e.g., cardiovascular disease, cancer, degenerative musculoskeletal disease). This modernization contributes to a significant increase in the burden of traumatic injuries as well, such as from road traffic crashes or industrial "accidents." This increase will continue for the foreseeable future: Trafficrelated deaths and injuries are forecast to increase by $80 \%$ in LMICs this decade [3]. Additionally, more than 500,000 women in LMICs die each year during childbirth, a tragedy practically unknown in the developed world nowadays [4].

The ability to meet the increasing need for surgical care is not keeping up with the pace. It has been estimated that around $11 \%$ of the world's disability-adjusted life-years (DALYs) are the result of surgical conditions [5]. This most certainly underestimates the situation in LMICs, as suggested by a study in Ghana showing that mortality alone from preventable or treatable surgical conditions accounted for $10.9 \%$ of all DALYs [6]. Factoring in the avoidable disability from surgical treatment would undoubtedly increase this figure significantly.

In most LMICs, access to basic surgical and anesthesia care is restricted, particularly for the poor and vulnerable populations in both urban and rural settings. Only a small percentage of patients who require operations are treated because of various constraints, including the dearth of human resources and capacities, inadequate logistics, 
inability to pay, obsolete infrastructure, lack of transportation and late presentation, unavailability of basic materials and supplies, lack of electricity, and erratic water supply systems.

Two recent analyses, utilizing the World Health Organization (WHO) Integrated Management for Emergency and Essential Surgical Care (IMEESC) toolkit [7] to assess the provision of surgical care in LMICs [8, 9], confirmed the enormous shortfalls in infrastructure, supplies, and procedures at district-level health facilities. Moreover, evaluations of trauma care resources in these countries highlight the gap between resource allocation and needs, which should be addressed through clear policies to prioritize remote areas and to allocate resources based on reliable injury data [10].

An important component of the gap between need and provision of care is insufficient human capacity at all levels: doctors, nurses, technicians, administrators. This problem has been accentuated by a significant brain drain not only from developing to developed countries but also within the countries themselves from rural to urban settings because of better financial, professional, and social incentives [11]. Of the 47 countries in sub-Saharan Africa, 38 do not meet the WHO recommended minimum of 20 doctors and 100 nurses per 100,000 population [12]; and this does not address the lack of specialties mix that is required to deal with the burden of diseases in sub-Saharan Africa or to meet the needs for surgical care.

Even when qualified providers are available, other barriers may exist: (1) lack of incentive for workers who are paid the same regardless of output; and (2) lack of material and supplies (e.g., internal fixation devices) because they are too expensive for the patient's ability to pay. In fact, the ability to pay for diagnostic tests or treatment often plays a determinant role in patient prioritization. Fortunately, the long-held argument that "surgery is too expensive for these places" is increasingly challenged by new data suggesting that appropriate surgical care can in fact be quite costeffective [13, 14]. Furthermore, it is now widely accepted that surgery is an essential component for achieving the reknown Millennium Development Goals, particularly those that address alleviation of poverty, poor maternal health, and some infectious diseases (e.g., human immunodeficiency virus infection/acquired immunodeficiency syndrome, or HIV/AIDS) [7].

\section{Current solutions}

How can the surgical skills gap of LMICs be filled, at least partially, to enable them to deal with the enormous DALYs they face? Approaches adopted so far have included (1) brief visits by surgeons from advantaged countries with a lot of donated equipment for a few highly technical operations that cannot be reproduced or maintained in the regions visited; (2) trainee residents being sent to spend time in a developing country as part of their training; and (3) ships weighing anchor offshore and offering some limited on-shore and/or on-board services.

Visiting surgeons and the surgical residents or trainees are often not equipped to deal with the broad spectrum of surgical needs and challenging conditions of the areas they visit. They are also often insensitive to or misinformed about the cultural attitudes of the indigenous people regarding disease and death. This calls for proper training programs to be organized for surgeons and anesthetists intending to work in culturally different and deprived low-income countries. Pinpoint provision of surgical care, mostly by nongovernmental organizations (NGOs) of all sizes and creeds, rarely contributes significantly to a transfer of skills and knowledge, both of which are to some extent irrelevant if the necessary technology is unavailable. Training abroad is rarely appropriate for the material and technical environments to which the trainee will return and is a major contributor to the brain drain noted earlier. Indeed, common mistakes and concerns about surgical humanitarian missions have been correctly analyzed elsewhere [15]. Failure to match technology to local needs and abilities, failure of NGOs to cooperate with each other and with local governments, failure to have a surgical follow-up plan, and insensitivity to local customs and beliefs are the most frequent and significant drawbacks of these missions, despite the fact that they stem from noble motives.

\section{Proposed solutions}

Local training programs might be the best option for addressing these challenges. These teaching and training opportunities should be offered not only to surgeons and anesthesiologists but also to general local medical and paramedical personnel in a way that supports and encourages appropriate and sustainable surgical care. Twinning between medical school hospitals in developed countries and those in the developing world has been proposed as one of the options for training international and local staff [16]. There can be several aspects to twinning, including close interdepartment/interdiscipline associations. There can also be interhospital twinning for the purposes of providing service, training, transfer of knowledge and technology, and research. It is a good concept that, when carefully considered and implemented, may be mutually beneficial and provide a sustainable and lasting solution to the unmet health needs of the developing world. Curriculum content for the training of surgeons must include 
general surgery, urology, orthopedics, obstetrics/gynecology, emergency care, and anesthesiology. The outcome of such a training program should be to make the surgeon reasonably self-sufficient and able to cope with most of the surgical problems of a district hospital in the developing world.

Local staff members who may benefit from such training may have to be encouraged to stay at the post with some form of inducement. Usually, resources are severely restricted in low-income countries, and trained personnel may not be adequately remunerated for them to want to stay in that situation. For a limited period, and to allow a certain kind of work ethic and institutional culture to evolve, it may be necessary to use some of the donor budgetary-support money, usually provided for some of these low-income countries on humanitarian grounds, to reward the trained local staff.

Accordingly, the role of governments or professionals and their associations to address training and teaching of surgical care in their own country cannot be underestimated. Efforts to promote wider access to surgical services would be aided by developing a consensus as to which surgical conditions warrant the most focused attention and investment. Those conditions with the highest public health burden and for which procedures are highly successful and feasible even in the most resource-constrained environments should be the main focus of governmental efforts. In some countries, nonphysicians have been trained to perform relatively simple surgical procedures. Compared with physician programs, in rural areas these programs are costeffective and have favorable outcomes.

An interesting project with encouraging and promising results has been started in Niger [17] where surgical procedures are performed in district hospitals by general practitioners who received 12 months of training in basic surgery. This was also of interest to these physicians because this certificate of completion provided a bonus to their annual salary in addition to the indemnity they receive as an incentive for working in rural areas. In terms of retention, all physicians trained from this program have so far remained at their posts. Specialized surgeons had the responsibility to (1) follow up on the outcomes from the generalists they trained and (2) provide continuous education and supervision during short stays in the district hospitals. Similar initiatives have been successfully implemented in other countries, including Mozambique, Burkina Faso, and Sudan.

Although the problem is enormous, the engagement of the political leaders together with development partners to relieve the burden of surgical disease is strategically important for a longer-term solution. Aggressive retention policies, such as improving the remuneration and working conditions of health workers, should be strongly encouraged by the governments and supported by their donor partners.

\section{Conclusions}

Strategies to deal with the unmet surgical needs of the developing world should include short-term humanitarian assistance, as is currently being offered, as well as longterm well-considered programs for training and retention of local staff. Surgeons intending to work in the developing world should undergo special training to enable them to deal with the peculiar surgical problems and the sociocultural and economic circumstances of the area they intend to visit. The benefits of globalization and the achievement of the Millennium Development Goals will be a mirage unless strategies are devised to deal with the disease burden of low-income countries, including surgical DALYs.

Open Access This article is distributed under the terms of the Creative Commons Attribution Noncommercial License which permits any noncommercial use, distribution, and reproduction in any medium, provided the original author(s) and source are credited.

\section{References}

1. Contini S (2007) Surgery in developing countries: why and how to meet surgical needs worldwide. Acta Biomed 78:4-5

2. King M, Bewes P, Cairns J et al (2003) Background to surgery. In: Primary surgery, vol 1. Oxford University Press, Oxford

3. Peden M, Scurfield R, Sleet D et al (eds) (2004) WHO world report on road traffic injuries prevention. World Health Organization, Geneva

4. Farmer PE, Kim YJ (2008) Surgery and global health: a view from beyond the OR. World J Surg 32:533-536

5. Debas HT, Gosselin RA, McCord C et al (2006) Surgery. In: Jamison DT, Breman JG, Measham AR et al (eds) Disease control priorities in developing countries, 2nd edn. Oxford University Press and World Bank, New York, pp 1245-1259

6. Duda RB, Hill AG (2007) Surgery in developing countries: should surgery have a role in population-based health care? Bull Am Coll Surg 78(5):13-18

7. World Health Organization (WHO) (2009) Emergency and essential surgical care: Global Initiative for Emergency and Essential Surgical Care (GIEESC). Available at www.who.int/ surgery. Accessed 31 Jan 2009

8. Contini S, Taqdeer A, Cherian M et al (2010) Emergency and essential surgical services in Afghanistan: still a missing challenge. World J Surg 34:473-478

9. Kushner AL, Cherian MN, Noel L et al (2010) Addressing the millennium development goals from a surgical perspective. Arch Surg 145:154-159

10. Nakahara S, Saint S, Sann S et al (2009) Evaluation of trauma care resources in health centers and referral hospitals in Cambodia. World J Surg 33:874-885

11. Johna S (2006) The rural surgeon: an endangered species. World J Surg 30:267-268

12. Hagopian A, Thompson MJ, Fordyce M et al (2004) The migration of physicians from sub-Saharan Africa to the United 
States of America: measures of the African brain drain. Hum Resour Health 2:17-22

13. Gosselin RA, Thind A, Bellardinelli A (2006) Cost/DALY averted in a small hospital in Sierra Leone: what is the contribution of different services? World J Surg 30:505-511

14. Gosselin RA, Heitto M (2008) Cost-effectiveness of a district trauma hospital in Battambang, Cambodia. World J Surg 32: $2450-2453$
15. Welling DR, Ryan JM, Burris DG et al (2010) Seven sins of humanitarian medicine. World J Surg 34:466-470

16. Riviello R, Ozgediz D, Renee Y et al (2010) Role of collaborative academic partnerships in surgical training, education, and provision. World J Surg 34:459-465

17. Sani R, Nameoua B, Yahaya A et al (2009) The impact of launching surgery at the district level in Niger. World J Surg 33: 2063-2068 